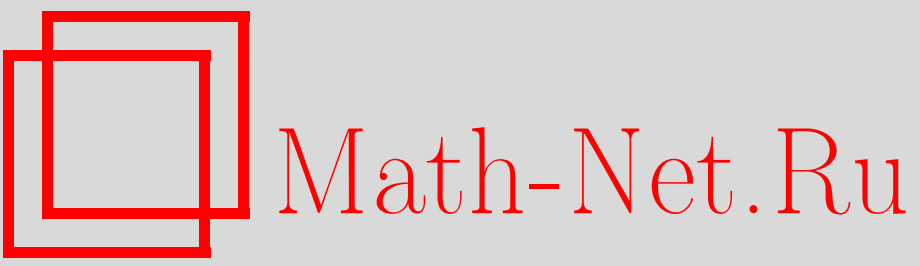

Г. В. Перельман, K вопросу о справедливости формулы Ито для разрывной функции, Теория вероятн. и ее примен., 2011, том 56, выпуск 3, 478-493

DOI: https://doi.org/10.4213/tvp4403

Использование Общероссийского математического портала Math-Net.Ru подразумевает, что вы прочитали и согласны с пользовательским соглашением

http://www.mathnet.ru/rus/agreement

Параметры загрузки:

IP: 54.198 .55 .26

26 апреля 2023 г., 15:48:03 
2011

(c) 2011 г.

\author{
ПЕРЕЛЬМАН Г. В.*
}

\title{
К ВОПРОСУ О СПРАВЕДЛИВОСТИ ФОРМУЛЫ ИТО ДЛЯ РАЗРЫВНОЙ ФУНКЦИИ ${ }^{1)}$
}

\begin{abstract}
В работе получены два обобщения классической формулы Ито для броуновского движения: получена формула, справедливая для функций, имеющих разрыв производной вдоль гладкой кривой, а также формула, справедливая для функций, имеющих разрыв вдоль монотонной гладкой кривой.
\end{abstract}

Ключевые слова и фразы: формула Ито, броуновское движение, винеровский процесс, дискретная формула Ито, формула Танака.

1. Введение. Рассмотрим различные известные варианты и обобщения формулы Ито.

Классическая формула Ито для функции из $C^{1,2}\left(\mathbf{R}_{+} \times \mathbf{R}\right)$ и броуновского движения выглядит следующим образом.

Предложение 1 (Ито [7]). Пусть $F \in C^{1,2}\left(\mathbf{R}_{+} \times \mathbf{R}\right), W_{t}-$ стандартное броуновское движение. Тогда

$$
\begin{aligned}
F\left(t, W_{t}\right)-F(0,0)= & \int_{0}^{t} \frac{\partial F}{\partial t}\left(s, W_{s}\right) d s+\int_{0}^{t} \frac{\partial F}{\partial x}\left(s, W_{s}\right) d W_{s} \\
& +\frac{1}{2} \int_{0}^{t} \frac{\partial^{2} F}{\partial x^{2}}\left(s, W_{s}\right) d s .
\end{aligned}
$$

В связи с тем что формула является одной из ключевых в теории стохастического исчисления, большое число работ направлены на обобщение классического результата, связанное с расширением классов функций и процессов, к которым она применима.

Большое значение имеет формула Танака, в которой выписывается аналогичная формула для недифференцируемой функции модуль.

Предложение 2 (Формула Танака).

$$
\left|W_{t}-a\right|=\left|W_{0}-a\right|+\int_{0}^{t} \operatorname{sign}\left(W_{s}-a\right) d W_{s}+L_{t}^{a}
$$

где $L_{t}^{a}=\lim _{\varepsilon \downarrow 0}(2 \varepsilon)^{-1} \int_{0}^{t} I\left(\left|W_{s}-a\right| \leqslant \varepsilon\right) d s$.

\footnotetext{
* Московский государственный университет им. М.В.Ломоносова, механикоматематический факультет, Ленинские горы, 119991 Москва, Россия; e-mail: glebperelman@gmail.com

1) Работа выполнена при поддержке РФФИ (грант № 10-08-01070_а).
} 
Ниже приведены примеры, последовательно обобщающие класс допустимых функций.

Предложение 3 (Ито-Танака-Мейер [8]). Пусть $F$ дифференцируема и $F^{\prime}(x)$ имеет ограниченную вариачию. Тогда

$$
F\left(W_{t}\right)=F\left(W_{0}\right)+\int_{0}^{t} F^{\prime}\left(W_{s}\right) d W_{s}+\frac{1}{2} \int_{\mathbf{R}} L_{t}^{a} F^{\prime \prime}(d a) .
$$

Предложение 4 (Було-Йор [9]). Пусть F дифференцируема и $F^{\prime}(x)$ локально ограниченна. Тогда

$$
F\left(W_{t}\right)=F\left(W_{0}\right)+\int_{0}^{t} F^{\prime}\left(W_{s}\right) d W_{s}-\frac{1}{2} \int_{\mathbf{R}} F^{\prime}(a) d_{a} L_{t}^{a} .
$$

Предложение 5 (Фёлмер-Проттер-Ширяев [4]). Пусть F дифферениируема и $F^{\prime} \in L_{\mathrm{loc}}^{2}$. Тогда

$$
F\left(W_{t}\right)=F\left(W_{0}\right)+\int_{0}^{t} F^{\prime}\left(W_{s}\right) d W_{s}+\frac{1}{2}[F(W), W]_{t},
$$

где $[F(W), W]_{t}=\mathbf{P}-\lim _{n \rightarrow \infty} \sum_{k}\left(F^{\prime}\left(W_{t_{k+1}^{n} \wedge t}\right)-F^{\prime}\left(W_{t_{k}^{n} \wedge t}\right)\right)\left(W_{t_{k+1}^{n} \wedge t}-W_{t_{k}^{n} \wedge t}\right)$ $u \sup _{k}\left(t_{k+1}^{n} \wedge t-t_{k}^{n} \wedge t\right) \rightarrow 0$.

Также имеются результаты Н. Айзенбаум [10] и А. С. Черного [5] для абсолютно непрерывных функций, на которые наложены определенные условия (касающиеся существования некоторых интегралов).

Наиболее близким к результатам настоящей работы является результат Пешкира, полученный в 2002 г.:

Предложение 6 (Пешкир [2]). Пусть $X_{t}$ - непрерьввньй семимартингал, $b: \mathbf{R}_{+} \rightarrow \mathbf{R}-$ функиия ограниченной вариачии,

$$
C=\left\{(s ; x) \in \mathbf{R}_{+} \times \mathbf{R} \mid x<g(s)\right\}, \quad D=\left\{(s ; x) \in \mathbf{R}_{+} \times \mathbf{R} \mid x>g(s)\right\}
$$

и непрерывная функиия $F: \mathbf{R}_{+} \times \mathbf{R} \rightarrow \mathbf{R}$ является функцией класса $C^{1,2}$ на замькканиях множеств $C$ и $D$.

Тогда

$$
\begin{aligned}
F\left(t, X_{t}\right)= & F\left(0, X_{0}\right)+\int_{0}^{t} F_{t}\left(s, X_{s}-\right) d s+\int_{0}^{t} F_{x}\left(s, X_{s}-\right) d X_{s} \\
& +\frac{1}{2} \int_{0}^{t} F_{x x}\left(s, X_{s}\right) I\left(X_{s} \neq b(s)\right) d\langle X, X\rangle_{s} \\
& +\frac{1}{2} \int_{0}^{t}\left(F_{x}\left(s, X_{s}+\right)-F_{x}\left(s, X_{s}-\right)\right) I\left(X_{s}=b(s)\right) d \ell_{s}^{b},
\end{aligned}
$$

где $\ell_{s}^{b}$ - локальное время $X_{t}$ на кривой $b(t)$ :

$$
\ell_{s}^{b}=\mathbf{P}-\lim _{\varepsilon \downarrow 0} \frac{1}{\varepsilon} \int_{0}^{s} I\left(b(r) \leqslant X_{r}<b(r)+\varepsilon\right) d\langle X, X\rangle_{r} .
$$


В данной работе получено два результата, близких к результату, сформулированному в предложении 6 . Первый результат является более слабым вариантом. Второй применим к более общему классу функций функции могут иметь разрыв по кривой (в работе [2] и в первой части настоящей работы допускаются только разрывы производных).

\section{2. Случай непрерывной функции.}

Теорема 1. Пусть функиия $g \in C^{1}, g(0) \neq 0$, является границей множества $A$ в $\mathbf{R}^{2}, F$ непрерьвна и имеет вид

$$
F(t, x)=\left\{\begin{array}{l}
f(t, x) \in C^{1,2}(\bar{A}), \\
\phi(t, x) \in C^{1,2}\left(\overline{\mathbf{R}_{+} \times \mathbf{R} \backslash A}\right) .
\end{array}\right.
$$

Тогда

$$
\begin{aligned}
F( & \left(W_{t}\right)-F(0,0) \\
= & \int_{0}^{t} \frac{\partial F}{\partial t}\left(s, W_{s}\right) I\left(W_{s} \neq g(s)\right) d s+\int_{0}^{t} \frac{\partial F}{\partial x}\left(s, W_{s}\right) I\left(W_{s} \neq g(s)\right) d W_{s} \\
& +\frac{1}{2} \int_{0}^{t} \frac{\partial^{2} F}{\partial x^{2}}\left(s, W_{s}\right) I\left(W_{s} \neq g(s)\right) d s \\
& +\lim _{\varepsilon \downarrow 0} \frac{1}{2 \varepsilon} \int_{0}^{t} \frac{F_{x+}^{\prime}(s, g(s))-F_{x-}^{\prime}(s, g(s))}{2} I\left(\left|W_{s}-g(s)\right|<\varepsilon\right) d s, \quad(1)
\end{aligned}
$$

если последний предел существует.

Здесь и далее

$$
\begin{gathered}
F_{t}^{\prime}=\frac{\partial F}{\partial t}, \quad F_{x}^{\prime}=\frac{\partial F}{\partial x}, \quad F_{x x}^{\prime \prime}=\frac{\partial^{2} F}{\partial x^{2}}, \\
F_{x+}^{\prime}(s, g(s))=\lim _{t \rightarrow s+0} F_{x}^{\prime}(t, g(s)), \quad F_{x-}^{\prime}(s, g(s))=\lim _{t \rightarrow s-0} F_{x}^{\prime}(t, g(s)) .
\end{gathered}
$$

Д ок а з а т е ль с т в о. Рассмотрим тождество, которое также иногда называют дискретной формулой Ито для симметричного случайного блуждания $Z_{t}$ :

$$
\begin{aligned}
F(t+1, & \left.Z_{t+1}\right)-F\left(t, Z_{t}\right)=F\left(t+1, Z_{t}\right)-F\left(t, Z_{t}\right) \\
& +\frac{F\left(t+1, Z_{t}+1\right)-F\left(t+1, Z_{t}-1\right)}{2}\left(Z_{t+1}-Z_{t}\right) \\
& +\frac{F\left(t+1, Z_{t}+1\right)-2 F\left(t+1, Z_{t}\right)+F\left(t+1, Z_{t}-1\right)}{2} .
\end{aligned}
$$

В справедливости этой формулы легко убедиться простой подстановкой обоих возможных случаев: $Z_{t+1}=Z_{t}+1$ и $Z_{t+1}=Z_{t}-1$.

Заметим, что достаточно доказать формулу (1) для функции $F$ с компактным носителем и для фиксированного $t$. Введем приближение 
броуновского движения случайным блужданием. Для натурального $n$ определим следующие моменты:

$$
\begin{aligned}
\tau_{n, 0} & :=0 \\
\tau_{n, i} & :=\inf \left\{s>\tau_{n, i-1}:\left|W_{s}-W_{\tau_{n, i-1}}\right|=\frac{\sqrt{t}}{2^{n}}\right\}, \quad i=1,2, \ldots
\end{aligned}
$$

В силу строго марковского свойства броуновского движения эти моменты будут независимы и одинаково распределены.

Вспомним, что процессы $W_{s}, W_{s}^{2}-s$ и $W_{s}^{4}-6 s W_{s}^{2}+3 s^{2}-$ мартингалы.

По теореме Дуба о преобразовании свободного выбора (Doob's optional sampling theorem) процессы

$$
\begin{gathered}
W_{s \wedge \tau_{n, 1}}, \quad W_{s \wedge \tau_{n, 1}}^{2}-\left(s \wedge \tau_{n, 1}\right), \\
W_{s \wedge \tau_{n, 1}}^{4}-6\left(s \wedge \tau_{n, 1}\right) W_{s \wedge \tau_{n, 1}}^{2}+3\left(s \wedge \tau_{n, 1}\right)^{2}
\end{gathered}
$$

также являются мартинагалами. Отсюда следует, что

$$
\begin{gathered}
\mathbf{E} W_{s \wedge \tau_{n, 1}}=0, \quad \mathbf{E} s \wedge \tau_{n, 1}=\mathbf{E} W_{s \wedge \tau_{n, 1}}^{2} \\
\mathbf{E}\left(s \wedge \tau_{n, 1}\right)^{2}=-\frac{1}{3} \mathbf{E} W_{s \wedge \tau_{n, 1}}^{4}+2 \mathbf{E}\left[\left(s \wedge \tau_{n, 1}\right) W_{s \wedge \tau_{n, 1}}^{2}\right] .
\end{gathered}
$$

Устремляя $s$ к бесконечности, получаем:

$$
\mathbf{E} W_{\tau_{n, 1}}=0, \quad \mathbf{E} \tau_{n, 1}=\frac{t}{2^{2 n}}, \quad \mathbf{E}\left(\tau_{n, 1}\right)^{2}=\frac{5}{3} \frac{t^{2}}{2^{4 n}} .
$$

Значит, $\tau_{n, i}-i t / 2^{2 n}-$ мартингал. Отсюда, из (3) и из неравенства Дуба для субмартингалов следует, что

$$
\mathbf{P}\left[\sup _{1 \leqslant i \leqslant 2^{2 n}}\left|\tau_{n, i}-i \frac{t}{2^{2 n}}\right|>\varepsilon\right] \leqslant \varepsilon^{-2} \mathbf{E}\left(\tau_{n, 2^{2 n}}-t\right)^{2}=\varepsilon^{-2} \frac{2}{3} \frac{t^{2}}{2^{2 n}} .
$$

Применяя лемму Бореля-Кантелли, получаем

$$
\mathbf{P}\left[\lim _{n \rightarrow \infty} \sup _{1 \leqslant i \leqslant 2^{2 n}}\left|\tau_{n, i}-i \frac{t}{2^{2 n}}\right|=0\right]=1
$$

Из равенства (5) и равномерной непрерывности функции, определенной на компактном интервале, следует, что

$$
\mathbf{P}\left[\lim _{n \rightarrow \infty} \sup _{1 \leqslant i \leqslant 2^{2 n}}\left|W_{\tau_{n, i}}-W_{i t / 2^{2 n}}\right|=0\right]=1
$$


Используя дискретную формулу Ито (2) и дискретную аппроксимацию броуновского движения, получаем

$$
\begin{aligned}
& F\left(\tau_{n, 2^{2 n}}, W_{\tau_{n, 2^{2 n}}}\right)-F(0,0)=\sum_{i=0}^{2^{2 n}-1}\left(F\left(\tau_{n, i+1}, W_{\tau_{n, i}}\right)-F\left(\tau_{n, i}, W_{\tau_{n, i}}\right)\right) \\
& +\sum_{i=0}^{2^{2 n}-1} \frac{F\left(\tau_{n, i+1}, W_{\tau_{n, i}}+\sqrt{t} / 2^{n}\right)-F\left(\tau_{n, i+1}, W_{\tau_{n, i}}-\sqrt{t} / 2^{n}\right)}{2 \sqrt{t} / 2^{n}} \\
& \quad \times\left(W_{\tau_{n, i+1}}-W_{\tau_{n, i}}\right) \\
& +\frac{1}{2} \sum_{i=0}^{2^{2 n}-1}\left(F\left(\tau_{n, i+1}, W_{\tau_{n, i}}+\frac{\sqrt{t}}{2^{n}}\right)-2 F\left(\tau_{n, i+1}, W_{\tau_{n, i}}\right)\right. \\
& \left.+F\left(\tau_{n, i+1}, W_{\tau_{n, i}}-\frac{\sqrt{t}}{2^{n}}\right)\right) .
\end{aligned}
$$

Левая часть равенства согласно (5) и (6) стремится к $F\left(t, W_{t}\right)-$ $F(0,0)$. Рассмотрим правую часть.

Пусть $A_{n}$ обозначает первое слагаемое в правой части (7). Тогда

$$
\begin{aligned}
A_{n} & =\sum_{i=0}^{2^{2 n}-1}\left(F\left(\tau_{n, i+1}, W_{\tau_{n, i}}\right)-F\left(\tau_{n, i}, W_{\tau_{n, i}}\right)\right) \\
& =\sum_{i=0}^{2^{2 n}-1} \frac{F\left(\tau_{n, i+1}, W_{\tau_{n, i}}\right)-F\left(\tau_{n, i}, W_{\tau_{n, i}}\right)}{\tau_{n, i+1}-\tau_{n, i}}\left(\tau_{n, i+1}-\tau_{n, i}\right) .
\end{aligned}
$$

Обозначим множество тех $i$, для которых отрезок, соединяющий точки $\left(\tau_{n, i+1}, W_{\tau_{n, i}}\right)$ и $\left(\tau_{n, i}, W_{\tau_{n, i}}\right)$, не пересекает границу $g(t)$ через $U_{1}^{n}$, а множество оставшихся через $U_{2}^{n}$. Тогда

$$
A_{n}=\sum_{U_{1}^{n}} F_{t}^{\prime}\left(\xi_{i}, W_{\tau_{n, i}}\right)\left(\tau_{n, i+1}-\tau_{n, i}\right)+\sum_{U_{2}^{n}}\left(F\left(\tau_{n, i+1}, W_{\tau_{n, i}}\right)-F\left(\tau_{n, i}, W_{\tau_{n, i}}\right)\right),
$$

где $\xi_{i}$ - точка на интервале $\left(\tau_{n, i}, \tau_{n, i+1}\right)$ из теоремы Лагранжа.

Рассмотрим сумму по $U_{2}^{n}$. Воспользовавшись равномерной непрерывностью функции, непрерывной на компакте, получаем

$$
\begin{aligned}
& \sum_{U_{2}^{n}}\left(F\left(\tau_{n, i+1}, W_{\tau_{n, i}}\right)-F\left(\tau_{n, i}, W_{\tau_{n, i}}\right)\right)<\sum_{U_{2}^{n}} c\left(\tau_{n, i+1}-\tau_{n, i}\right) \\
& \quad \leqslant c \max _{i}\left(\tau_{n, i+1}-\tau_{n, i}\right) \cdot \#\left\{i \in U_{2}^{n}\right\} \sim c t \frac{\#\left\{i \in U_{2}^{n}\right\}}{2^{2 n}} \rightarrow 0
\end{aligned}
$$

(здесь \# $\# \cdot\}$ - количество элементов в множестве).

Рассмотрим сумму по $U_{1}^{n}$ :

$\sum_{U_{1}^{n}} F_{t}^{\prime}\left(\tau_{n, i}, W_{\tau_{n, i}}\right)\left(\tau_{n, i+1}-\tau_{n, i}\right)+\sum_{U_{1}^{n}}\left(F_{t}^{\prime}\left(\xi_{i}, W_{\tau_{n, i}}\right)-F_{t}^{\prime}\left(\tau_{n, i}, W_{\tau_{n, i}}\right)\right)\left(\tau_{n, i+1}-\tau_{n, i}\right)$. 
Воспользуемся тем, что функция $F_{t}^{\prime}$, непрерывная на каждой из двух областей непрерывности, равномерно непрерывна на них. Пусть $c_{j}, j=1,2$, - константы из условия равномерной непрерывности для этих областей. Если мы выберем $c=\max \left\{c_{1}, c_{2}\right\}$, то для любых двух точек из одной области непрерывности (не важно для какой)

$$
\left|F\left(t_{1}, W_{t_{1}}\right)-F\left(t_{2}, W_{t_{2}}\right)\right|<c\left|t_{1}-t_{2}\right| .
$$

Точки $\tau_{n, i}$ и $\xi_{i}$ для $i \in U_{1}^{n}$ находятся в одной области непрерывности, поэтому, воспользовавшись последним неравенством, получим

$$
\begin{aligned}
& \left|\sum_{U_{1}^{n}}\left(F_{t}^{\prime}\left(\xi_{i}, W_{\tau_{n, i}}\right)-F_{t}^{\prime}\left(\tau_{n, i}, W_{\tau_{n, i}}\right)\right)\left(\tau_{n, i+1}-\tau_{n, i}\right)\right| \\
& \quad<\sum_{U_{1}^{n}} c\left(\xi_{i}-\tau_{n, i}\right)\left(\tau_{n, i+1}-\tau_{n, i}\right)<c t \max _{i}\left(\tau_{n, i+1}-\tau_{n, i}\right),
\end{aligned}
$$

что стремится к нулю согласно (5).

Рассмотрим $\sum_{U_{1}^{n}} F_{t}^{\prime}\left(\tau_{n, i}, W_{\tau_{n, i}}\right)\left(\tau_{n, i+1}-\tau_{n, i}\right)$. Выберем $\varepsilon>0$. Тогда согласно (5) существует такое $N$, что для любого $n>N$

$$
\begin{aligned}
\sum_{U_{1}^{n}} F_{t}^{\prime}\left(\tau_{n, i}, W_{\tau_{n, i}}\right)\left(\tau_{n, i+1}-\tau_{n, i}\right) I\left(\rho\left(\left(\tau_{n, i}, W_{\tau_{n, i}}\right), g\right)>\varepsilon\right) \\
=\sum_{i=1}^{2^{2 n}-1} F_{t}^{\prime}\left(\tau_{n, i}, W_{\tau_{n, i}}\right)\left(\tau_{n, i+1}-\tau_{n, i}\right) I\left(\rho\left(\left(\tau_{n, i}, W_{\tau_{n, i}}\right), g\right)>\varepsilon\right),
\end{aligned}
$$

где $\rho$ есть расстояние от точки до графика.

Последняя сумма есть риманова сумма для интеграла $\int_{0}^{t} F_{t}^{\prime}\left(s, B_{s}\right) \times$ $I\left(\rho\left(\left(s, B_{s}\right), g\right)>\varepsilon\right) d s$.

Устремляя $n$ к бесконечности и $\varepsilon$ к нулю, получаем

$$
\sum_{U_{1}^{n}} F_{t}^{\prime}\left(\tau_{n, i}, W_{\tau_{n, i}}\right)\left(\tau_{n, i+1}-\tau_{n, i}\right) \rightarrow \int_{0}^{t} F_{t}^{\prime}\left(s, B_{s}\right) I\left(B_{s} \neq g(s)\right) d s .
$$

Перейдем ко второму слагаемому в правой части (7). Обозначим

$$
\begin{aligned}
H_{n}(s):= & \sum_{i=0}^{2^{2 n}-1} \frac{F\left(\tau_{n, i+1}, W_{\tau_{n, i}}+\sqrt{t} / 2^{n}\right)-F\left(\left(\tau_{n, i+1}, W_{\tau_{n, i}}-\sqrt{t} / 2^{n}\right)\right.}{2\left(\sqrt{t} / 2^{n}\right)} \\
& \times I_{\left(\tau_{n, i}, \tau_{n, i+1}\right]}(s), \\
K_{n}(s):= & \sum_{i=0}^{2^{2 n}-1} \frac{F\left(s, W_{\tau_{n, i}}+\sqrt{t} / 2^{n}\right)-F\left(s, W_{\tau_{n, i}}-\sqrt{t} / 2^{n}\right)}{2\left(\sqrt{t} / 2^{n}\right)} I_{\left(\tau_{n, i}, \tau_{n, i+1}\right]}(s) .
\end{aligned}
$$

Пусть $U_{3}^{n}$ обозначает множество тех $0 \leqslant i<2^{2 n}$, для которых график $x=g(t)$ не пересекает квадрат между точками $\left(\tau_{n, i}, W_{\tau_{n, i}}-\sqrt{t} / 2^{2 n}\right)$ и $\left(\tau_{n, i+1}, W_{\tau_{n, i}}+\sqrt{t} / 2^{2 n}\right)$. Множество оставшихся $i$ обозначим $U_{4}^{n}$. 
Используя введенные множества, разобьем $K_{n}(s)$ на две части:

$$
\begin{aligned}
K_{n}^{(1)}(s) & :=\sum_{U_{3}^{n}} \frac{F\left(s, W_{\tau_{n, i}}+\sqrt{t} / 2^{n}\right)-F\left(s, W_{\tau_{n, i}}-\sqrt{t} / 2^{n}\right)}{2\left(\sqrt{t} / 2^{n}\right)} I_{\left(\tau_{n, i}, \tau_{n, i+1}\right]}(s), \\
K_{n}^{(2)}(s) & :=\sum_{U_{4}^{n}} \frac{F\left(s, W_{\tau_{n, i}}+\sqrt{t} / 2^{n}\right)-F\left(s, W_{\tau_{n, i}}-\sqrt{t} / 2^{n}\right)}{2\left(\sqrt{t} / 2^{n}\right)} I_{\left(\tau_{n, i}, \tau_{n, i+1}\right]}(s) .
\end{aligned}
$$

Таким образом, второе слагаемое формулы (7) может быть переписано как $\int_{0}^{\tau_{n, 2^{2 n}}} H_{n}(s) d W_{s}$.

Выберем $\varepsilon>0$ и $\delta>0$. Имеем:

$$
\begin{aligned}
& p_{n}:=\mathbf{P}\left[\left|\int_{0}^{\tau_{n, 2}{ }^{2 n}} H_{n}(s) d W_{s}-\int_{0}^{t} F_{x}^{\prime}\left(s, W_{s}\right) I\left(W_{s} \neq g(s)\right) d W_{s}\right|>\delta\right] \\
& \leqslant \mathbf{P}\left[\left|\int_{0}^{\tau_{n, 2}{ }^{2 n}} K_{n} d W_{s}-\int_{0}^{\tau_{n, 2} 2^{2 n}} H_{n} d W_{s}\right|>\frac{\delta}{6}\right]+\mathbf{P}\left[\left|\int_{0}^{\tau_{n, 2}{ }^{2 n}} K_{n}^{(2)} d W_{s}\right|>\frac{\delta}{6}\right] \\
& +\mathbf{P}\left[\left\{\left|\int_{0}^{\tau_{n, 2^{2 n}}} K_{n}^{(1)}(s) d W_{s}-\int_{0}^{\tau_{n, 2^{2 n}}} F_{x}^{\prime}\left(s, W_{s}\right) I\left(W_{s} \neq g(s)\right) d W_{s}\right|>\frac{\delta}{3}\right\}\right. \\
& +2 \mathbf{P}\left[\left|\tau_{n, 2^{2 n}}-t\right|>\varepsilon\right]+\mathbf{P}\left[\left\{\left|\int_{t}^{\tau_{n, 2}{ }^{2 n}} F_{x}^{\prime}\left(s, W_{s}\right) I\left(W_{s} \neq g(s)\right) d W_{s}\right|>\frac{\delta}{3}\right\}\right. \\
& +\mathbf{P}\left[\left\{\left|\int_{t}^{\tau_{n, 2^{2 n}}} F_{x}^{\prime}\left(s, W_{s}\right) I\left(W_{s} \neq g(s)\right) d W_{s}\right|>\frac{\delta}{3}\right\} \cap\left\{-\varepsilon \leqslant t-\tau_{n, 2^{2 n}} \leqslant 0\right\}\right] \\
& \leqslant \mathbf{P}\left[\left|\int_{0}^{\tau_{n, 2^{2 n}}}\left(K_{n}-H_{n}\right) d W_{s}\right|>\frac{\delta}{6}\right]+\mathbf{P}\left[\left|\int_{0}^{\tau_{n, 2}^{2 n}} K_{n}^{(2)} d W_{s}\right|>\frac{\delta}{6}\right] \\
& +\mathbf{P}\left[\sup _{0 \leqslant \tau \leqslant t+\varepsilon}\left|\int_{0}^{\tau}\left(K_{n}^{(1)}(s)-F_{x}^{\prime}\left(s, W_{s}\right) I\left(W_{s} \neq g(s)\right)\right) d W_{s}\right|>\frac{\delta}{3}\right] \\
& +2 \mathbf{P}\left[\left|\tau_{n, 2^{2 n}}-t\right|>\varepsilon\right]+\mathbf{P}\left[\sup _{t \leqslant \tau \leqslant t+\varepsilon}\left|\int_{t}^{\tau} F_{x}^{\prime}\left(s, W_{s}\right) d W_{s}\right|>\frac{\delta}{3}\right] \\
& +\mathbf{P}\left[\left|\int_{t-\varepsilon}^{t} F_{x}^{\prime}\left(s, W_{s}\right) I\left(W_{s} \neq g(s)\right) d W_{s}\right|>\frac{\delta}{6}\right] \\
& +\mathbf{P}\left[\sup _{t-\varepsilon \leqslant \tau \leqslant t}\left|\int_{t-\varepsilon}^{\tau} F_{x}^{\prime}\left(s, W_{s}\right) I\left(W_{s} \neq g(s)\right) d W_{s}\right|>\frac{\delta}{6}\right] .
\end{aligned}
$$

Применяя к слагаемым предыдущего выражения максимальное неравенство для субмартингалов, неравенства Чебышёва, Иенсена и изометрию Интеграла Ито, получим

$$
p_{n} \leqslant \frac{6}{\delta} \mathbf{E}\left|\int_{0}^{\tau_{n, 2^{2 n}}}\left(K_{n}(s)-H_{n}(s)\right) d W_{s}\right|+\frac{6}{\delta} \mathbf{E}\left|\int_{0}^{\tau_{n, 2^{2 n}}} K_{n}^{(2)}(s) d W_{s}\right|
$$




$$
\begin{aligned}
+ & \frac{3}{\delta} \mathbf{E}\left|\int_{0}^{t+\varepsilon}\left(K_{n}(s)-F_{x}^{\prime}\left(s, W_{s}\right) I\left(W_{s} \neq g(s)\right)\right) d W_{s}\right|+\frac{2}{\varepsilon} \mathbf{E}\left|\tau_{n, 2^{2 n}}-t\right| \\
+ & \frac{3}{\delta} \mathbf{E}\left|\int_{t}^{t+\varepsilon} F_{x}^{\prime}\left(s, W_{s}\right) I\left(W_{s} \neq g(s)\right) d W_{s}\right| \\
+ & \frac{12}{\delta} \mathbf{E}\left|\int_{t-\varepsilon}^{t} F_{x}^{\prime}\left(s, W_{s}\right) I\left(W_{s} \neq g(s)\right) d W_{s}\right| \\
\leqslant & \frac{6}{\delta}\left(\mathbf{E}\left|\int_{0}^{\tau_{n, 2^{2 n}}}\left(K_{n}(s)-H_{n}(s)\right)^{2} d s\right|\right)^{1 / 2} \\
& +\frac{6}{\delta}\left(\mathbf{E}\left|\int_{0}^{\tau_{n, 2^{2 n}}}\left(K_{n}^{(2)}(s)\right)^{2} d s\right|\right)^{1 / 2} \\
& +\frac{3}{\delta}\left(\mathbf{E}\left|\int_{0}^{t+\varepsilon}\left(K_{n}^{(1)}(s)-F_{x}^{\prime}\left(s, W_{s}\right) I\left(W_{s} \neq g(s)\right)\right)^{2} d s\right|\right)^{1 / 2} \\
& +\frac{2}{\varepsilon}\left(\mathbf{E}\left(\tau_{n, 2^{2 n}}-t\right)^{2}\right)^{2}+\frac{3}{\delta}\left(\mathbf{E}\left|\int_{t}^{t+\varepsilon} F_{x}^{\prime}\left(s, W_{s}\right)^{2} I\left(W_{s} \neq g(s)\right) d s\right|\right)^{1 / 2} \\
& +\frac{12}{\delta}\left(\mathbf{E}\left|\int_{t-\varepsilon}^{t} F_{x}^{\prime}\left(s, W_{s}\right)^{2} I\left(W_{s} \neq g(s)\right) d s\right|\right)^{1 / 2} .
\end{aligned}
$$

Воспользовавшись ограниченностью $F_{x}^{\prime} \cdot I\left(W_{s} \neq g(s)\right)$ для последних двух слагаемых, равномерной непрерывностью $F$ (для разности $\left.K_{n}(s)-H_{n}(s)\right)$ и оценкой (4) (для четвертого слагаемого), получаем, что при стремлении $n$ к бесконечности, а $\varepsilon$ к нулю эти слагаемые будут стремиться к нулю. Второе слагаемое также стремится к нулю при $n$, стремящемся к бесконечности. Для доказательства воспользуемся равномерной ограниченностью $F$ (знаменатель сократится) и получим выражение, к которому можно применить рассуждения, использованные при работе с первым слагаемым суммы (7).

Осталось разобраться с третьим слагаемым в (8).

Применим к $K_{n}^{(1)}$ теорему Лагранжа (о среднем значении):

$$
\begin{aligned}
& \mathbf{E} \int_{0}^{t+\varepsilon}\left(K_{n}^{(1)}(s)-F_{x}^{\prime}\left(s, W_{s}\right) I\left(W_{s} \neq g(s)\right)\right)^{2} d s \\
& =\mathbf{E} \int_{0}^{t+\varepsilon}\left(\sum_{U_{3}^{n}} F_{x}^{\prime}\left(s, W_{\tau_{n, i}}+\theta \frac{\sqrt{t}}{2^{n}}\right) I\left(W_{s} \neq g(s)\right) I_{\left(\tau_{n, i}, \tau_{n, i+1}\right]}(s)\right. \\
& \left.-F_{x}^{\prime}\left(s, W_{s}\right) I\left(W_{s} \neq g(s)\right)\right)^{2} d s\left(\text { здесь } \theta=\theta\left(W_{\tau_{n, i}}\right) \text { и }|\theta|<1\right) \\
& =\sum_{U_{3}^{n}} \mathbf{E} \int_{0}^{t+\varepsilon}\left(F_{x}^{\prime}\left(s, W_{\tau_{n, i}}+\theta \frac{\sqrt{t}}{2^{n}}\right)-F_{x}^{\prime}\left(s, W_{s}\right)\right)^{2} I_{\left(\tau_{n, i}, \tau_{n, i+1}\right]}(s) d s \\
& +\sum_{U_{4}^{n}} \mathbf{E} \int_{0}^{t+\varepsilon} F_{x}^{\prime}\left(s, W_{s}\right)^{2} I\left(W_{s} \neq g(s)\right) I_{\left(\tau_{n, i}, \tau_{n, i+1}\right]}(s) d s \\
& +\mathbf{E} \int_{0}^{t+\varepsilon} F_{x}^{\prime}\left(s, W_{s}\right)^{2} I\left(W_{s} \neq g(s)\right) I_{\left(\tau_{n, 2^{2 n}}, \infty\right)}(s) d s
\end{aligned}
$$




$$
\begin{aligned}
& \leqslant \sum_{U_{3}^{n}} \mathbf{E}\left[\left(\sup _{\tau_{n, i} \leqslant s \leqslant \tau_{n, i+1}}\left|F_{x}^{\prime}\left(s, W_{\tau_{n, i}}+\theta \frac{\sqrt{t}}{2^{n}}\right)-F_{x}^{\prime}\left(s, W_{s}\right)\right|\right)^{2}\left(\tau_{n, i+1}-\tau_{n, i}\right)\right] \\
& +M^{2} \sum_{U_{4}^{n}} \mathbf{E} \int_{0}^{t+\varepsilon} I_{\left(\tau_{n, i}, \tau_{n, i+1}\right]}(s) d s+M^{2} \mathbf{E}\left|\tau_{n, 2^{2 n}}-t-\varepsilon\right| \\
& \quad \quad\left(\text { здесь } M-\text { максимум }\left|F_{x}^{\prime}\right| I\left(W_{s} \neq g(s)\right)\right) \\
& \leqslant \sum_{U_{3}^{n}}\left(\mathbf{E}\left(\sup _{\tau_{n, i} \leqslant s \leqslant \tau_{n}, i+1}\left|F_{x}^{\prime}\left(s, W_{\tau_{n, i}}+\theta \frac{\sqrt{t}}{2^{n}}\right)-F_{x}^{\prime}\left(s, W_{s}\right)\right|\right)^{4}\right. \\
& \left.\quad \times \mathbf{E}\left(\tau_{n, i+1}-\tau_{n, i}\right)^{2}\right)^{1 / 2} \\
& +M^{2} \sum_{U_{4}^{n}} \frac{t}{2^{2 n}}+M^{2} \mathbf{E}\left(\tau_{n, 2^{2 n}}-t\right)^{2}+M^{2} \varepsilon .
\end{aligned}
$$

При последнем переходе было использовано второе равенство в (3). Применяя третье равенство в (3), получаем, что последнее выражение не превосходит

$$
\begin{aligned}
& \sqrt{\frac{5}{3}} t\left(\mathbf { E } \left(\operatorname { s u p } \left\{\left|F_{x}^{\prime}(s, u)-F_{x}^{\prime}(s, v)\right|:|u-v|\right.\right.\right. \\
& \left.\left.\left.\leqslant \sup _{\substack{0 \leqslant i \leqslant 2^{2 n}-1 \\
\tau_{n, i} \leqslant s \leqslant \tau_{n, i+1}}}\left|W_{\tau_{n, i}}+\theta \frac{\sqrt{t}}{2^{n}}-W_{s}\right|, t \in \bigcup_{i \in U_{3}^{n}}\left(\tau_{n, i}, \tau_{n, i+1}\right)\right\}\right)^{4}\right)^{1 / 2} \\
& \quad+M^{2} t \frac{\#\left\{U_{4}^{n}\right\}}{2^{2 n}}+M^{2} \mathbf{E}\left(\tau_{n, 2^{2 n}}-t\right)^{2}+M^{2} \varepsilon .
\end{aligned}
$$

Согласно (5),

$$
\sup _{\substack{0 \leqslant i \leqslant 22^{2 n}-1 \\ \tau_{n, i} \leqslant s \leqslant \tau_{n, i+1}}}\left|s-\tau_{n, i}\right|=\sup _{0 \leqslant i \leqslant 2^{2 n}-1}\left|\tau_{n, i+1}-\tau_{n, i}\right| \leqslant 2 \sup _{\substack{1 \leqslant i \leqslant 2^{2 n} \\ 1}}\left|\tau_{n, i}-i \frac{t}{2^{2 n}}\right|+\frac{t}{2^{2 n}},
$$

последнее выражение стремится к нулю при $n$, стремящемся к бесконечности.

В силу равномерной непрерывности непрерывной функции, определенной на компакте, равномерной непрерывности $F_{x}^{\prime}$ на каждой из областей (и выбора максимального коэффициента), получаем

$$
\limsup _{n \rightarrow \infty}\left\{\left|F_{x}^{\prime}(s, u)-F_{x}^{\prime}(s, v)\right|:|u-v| \leqslant \sup _{\substack{0 \leqslant i \leqslant 2^{2 n}-1 \\ \tau_{n, i} \leqslant s \leqslant \tau_{n, i+1}}}\left|W_{\tau_{n, i}}+\theta \frac{\sqrt{t}}{2^{n}}-W_{s}\right|\right\}=0 .
$$

Таким образом, мы получили желаемое для третьего слагаемого в (8) и, следовательно, для второй суммы в (7).

Перейдем к рассмотрению третьей суммы в правой части (7) $\sum_{i=0}^{2^{2 n}-1}\left(F\left(\tau_{n, i+1}, W_{\tau_{n, i}}+\frac{\sqrt{t}}{2^{n}}\right)-2 F\left(\tau_{n, i+1}, W_{\tau_{n, i}}\right)+F\left(\tau_{n, i+1}, W_{\tau_{n, i}}-\frac{\sqrt{t}}{2^{n}}\right)\right)$. 
Аналогично тому, что мы делали для первого слагаемого в (7), пусть $U_{5}^{n}$ обозначает множество тех $i$, для которых отрезок, соединяющий точки $\left(\tau_{n, i+1}, W_{\tau_{n, i}}-\sqrt{t} / 2^{n}\right)$ и $\left(\tau_{n, i+1}, W_{\tau_{n, i}}+\sqrt{t} / 2^{n}\right)$, не пересекает границу $g(t)$, а $U_{6}^{n}$ - множество остальных $i$. Тем самым мы разбили сумму на две части (по соответствующим подмножествам).

Рассмотрим сумму по $U_{5}^{n}$ :

$$
\begin{aligned}
& \mid \sum_{U_{5}^{n}}\left(F\left(\tau_{n, i+1}, W_{\tau_{n, i}}+\frac{\sqrt{t}}{2^{n}}\right)-2 F\left(\tau_{n, i+1}, W_{\tau_{n, i}}\right)+F\left(\tau_{n, i+1}, W_{\tau_{n, i}}-\frac{\sqrt{t}}{2^{n}}\right)\right) \\
& \quad-\int_{0}^{t} F_{x x}^{\prime \prime}\left(s, W_{s}\right) I\left(W_{s} \neq g(s)\right) d s \mid \\
& \leqslant \mid \sum_{U_{5}^{n}}\left(F\left(\tau_{n, i+1}, W_{\tau_{n, i}}+\frac{\sqrt{t}}{2^{n}}\right)-2 F\left(\tau_{n, i+1}, W_{\tau_{n, i}}\right)\right. \\
& \left.\quad+F\left(\tau_{n, i+1}, W_{\tau_{n, i}}-\frac{\sqrt{t}}{2^{n}}\right)\right)-\sum_{U_{5}^{n}} F_{x x}^{\prime \prime}\left(\tau_{n, i+1}, W_{i t / 2^{2 n}}\right) \frac{t}{2^{2 n}} \mid \\
& \quad+\left|\sum_{U_{5}^{n}} F_{x x}^{\prime \prime}\left(\tau_{n, i+1}, W_{i t / 2^{2 n}}\right)\left(\frac{t}{2^{2 n}}-(\tau(n, i+1)-\tau(n, i))\right)\right| \\
& \quad+\left|\sum_{U_{5}^{n}} F_{x x}^{\prime \prime}\left(\tau_{n, i+1}, W_{i t / 2^{2 n}}\right) \frac{t}{2^{2 n}}-\int_{0}^{t} F_{x x}^{\prime \prime}\left(s, W_{s}\right) I\left(W_{s} \neq g(s)\right) d s\right| .
\end{aligned}
$$

Используя равномерную непрерывность, переходим от $F_{x x}^{\prime \prime}\left(\tau_{n, i+1}, W_{\tau_{n, i}}\right)$ к $F_{x x}^{\prime \prime}\left(\tau_{n, i}, W_{\tau_{n, i}}\right)$, после этого пользуемся интегрируемостью второй производной по Риману и получаем, что

$$
\sum_{U_{5}^{n}} F_{x x}^{\prime \prime}\left(\tau_{n, i+1}, W_{\tau_{n, i+1}}\right)\left(\tau_{n, i+1}-\tau_{n, i}\right) \rightarrow \int_{0}^{t} F_{x x}^{\prime \prime}\left(s, W_{s}\right) I\left(W_{s} \neq g(s)\right) d s
$$

(действуем так же, как при разборе аналогичной суммы первого слагаемого в правой части (7))

$$
\begin{aligned}
& \left|\sum_{U_{5}^{n}} F_{x x}^{\prime \prime}\left(\tau_{n, i+1}, W_{i t / 2^{2 n}}\right)\left(\frac{t}{2^{2 n}}-(\tau(n, i+1)-\tau(n, i))\right)\right| \\
& \quad \leqslant M\left|\sum_{U_{5}^{n}}\left(\frac{t}{2^{2 n}}-(\tau(n, i+1)-\tau(n, i))\right)\right| \leqslant M\left|t-\tau_{n, 2^{2 n}}\right| .
\end{aligned}
$$

Последнее выражение стремится к нулю (это доказано при разборе второго слагаемого суммы (7)).

$\mathrm{K}$ первому слагаемому в правой части (9) (обозначим его $R_{3}^{n}$ ) применим формулу Тейлора с остаточным членом в интегральной форме 
(остаточный член второго порядка, разложение по $x$ ):

$$
\begin{aligned}
R_{3}^{n} \leqslant \sum_{U_{5}^{n}} \mid & \int_{W_{\tau_{n, i}}}^{W_{\tau_{n, i}}+\sqrt{t} / 2^{n}} F_{x x}^{\prime \prime}\left(\tau_{n, i+1}, x\right)\left(W_{\tau_{n, i}}+\frac{\sqrt{t}}{2^{n}}-x\right) d x \\
& -\frac{1}{2} F_{x x}^{\prime \prime}\left(\tau_{n, i+1}, W_{\tau_{n, i}}\right) \frac{t}{2^{2 n}} \mid \\
+ & \sum_{U_{5}^{n}} \mid \int_{W_{\tau_{n, i}}}^{W_{\tau_{n, i}}-\sqrt{t} / 2^{n}} F_{x x}^{\prime \prime}\left(\tau_{n, i+1}, x\right)\left(W_{\tau_{n, i}}-\frac{\sqrt{t}}{2^{n}}-x\right) d x \\
& -\frac{1}{2} F_{x x}^{\prime \prime}\left(\tau_{n, i+1}, W_{\tau_{n, i}}\right) \frac{t}{2^{2 n}} \mid \\
+ & t \sup \left\{\left|F_{x x}^{\prime \prime}(s, u)-F_{x x}^{\prime \prime}(s, v)\right|:\right. \\
0 \leqslant & \left.u-v \leqslant \sup _{1 \leqslant i \leqslant 2^{2 n}}\left|W_{\tau_{n, i}}-W_{i t / 2^{2 n}}\right|, x \neq g(s) \forall x \in(u, v)\right\} .
\end{aligned}
$$

Заметим, что для действительных $x$ и $y$ и дважды непрерывнодифференцируемой функции $f$

$$
\left|\int_{x}^{y} f^{\prime \prime}(s)(y-s) d s-\frac{1}{2} f^{\prime \prime}(x)(y-x)^{2}\right| \leqslant(y-x)^{2} \sup _{|u-v| \leqslant|x-y|}\left|f^{\prime \prime}(u)-f^{\prime \prime}(v)\right| .
$$

Таким образом, получаем

$$
\begin{aligned}
R_{3}^{n} \leqslant & 2 t \sup \left\{\left|F_{x x}^{\prime \prime}(s, u)-F_{x x}^{\prime \prime}(s, v)\right|:|u-v| \leqslant \frac{\sqrt{t}}{2^{n}}, x \neq g(s) \forall x \in(u, v)\right\} \\
+ & t \sup \left\{\left|F_{x x}^{\prime \prime}(s, u)-F_{x x}^{\prime \prime}(s, v):\right| u-v\left|\leqslant \sup _{i \in U_{5}^{n}}\right| W_{\tau_{n, i}}-W_{i t / 2^{2 n}} \mid,\right. \\
& x \neq g(s) \forall x \in(u, v)\} .
\end{aligned}
$$

Согласно равномерной непрерывности второй производной в каждой из областей непрерывности и формуле (6), правая часть последнего неравенства стремится к нулю при $n$, стремящемся к бесконечности.

Для завершения доказательства рассмотрим оставшиеся слагаемые $\left(\right.$ по $\left.U_{6}^{n}\right)$ третьей суммы.

Заметим, что если у нас образовалась одна «критическая» тройка (у которой соответствующий отрезок пересекает границу областей), то образуется и вторая, отличающаяся от нее на одну точку. Иными словами, на плоскости две тройки образуют «критическую» четверку точек, имеющих по оси $t$ координату $\tau_{n, i+1}$, а по оси $x$ координаты $W_{\tau_{n, i}}-\sqrt{t} / 2^{n}$, $W_{\tau_{n, i}}, W_{\tau_{n, i}}+\sqrt{t} / 2^{n}$ и $W_{\tau_{n, i}}+2 \sqrt{t} / 2^{n}$. Индикаторы выбираем так, чтобы не считать образовавшиеся тройки по два раза (домножая выражение на 2). 
Преобразуем оставшиеся слагаемые:

$$
\begin{gathered}
\sum_{U_{6}^{n}}\left(F\left(\tau_{n, i+1}, W_{\tau_{n, i}}+\frac{\sqrt{t}}{2^{n}}\right)-2 F\left(\tau_{n, i+1}, W_{\tau_{n, i}}\right)+F\left(\tau_{n, i+1}, W_{\tau_{n, i}}-\frac{\sqrt{t}}{2^{n}}\right)\right) \\
=\sum_{i=0}^{2^{2 n}-2}\left(F\left(\tau_{n, i+1}, W_{\tau_{n, i}}+2 \frac{\sqrt{t}}{2^{n}}\right)-F\left(\tau_{n, i+1}, W_{\tau_{n, i}}+\frac{\sqrt{t}}{2^{n}}\right)\right. \\
\left.-F\left(\tau_{n, i+1}, W_{\tau_{n, i}}\right)+F\left(\tau_{n, i+1}, W_{\tau_{n, i}}-\frac{\sqrt{t}}{2^{n}}\right)\right) \\
\times I\left(0 \leqslant g\left(\tau_{n, i+1}\right)-W_{\tau_{n, i}}<\frac{1}{2} \frac{\sqrt{t}}{2^{n}}\right) \\
+\sum_{i=0}^{2^{2 n}-2}\left(F\left(\tau_{n, i+1}, W_{\tau_{n, i}}+2 \frac{\sqrt{t}}{2^{n}}\right)-F\left(\tau_{n, i+1}, W_{\tau_{n, i}}+\frac{\sqrt{t}}{2^{n}}\right)\right. \\
\left.-F\left(\tau_{n, i+1}, W_{\tau_{n, i}}\right)+F\left(\tau_{n, i+1}, W_{\tau_{n, i}}-\frac{\sqrt{t}}{2^{n}}\right)\right) \\
\times I\left(0 \leqslant W_{\tau_{n, i}}+\frac{\sqrt{t}}{2^{n}}-g\left(\tau_{n, i+1}\right)<\frac{1}{2} \frac{\sqrt{t}}{2^{n}}\right) .
\end{gathered}
$$

По теореме Лагранжа (о среднем значении) последнее выражение равно

$$
\begin{aligned}
\sum_{i=0}^{2^{2 n}-2}( & \left.F_{x}^{\prime}\left(\tau_{n, i+1}, \eta_{1, i}^{n}\right) \frac{\sqrt{t}}{2^{n}}-F_{x}^{\prime}\left(\tau_{n, i+1}, \eta_{2, i}^{n}\right) \frac{\sqrt{t}}{2^{n}}\right) \\
\times & I\left(0 \leqslant g\left(\tau_{n, i+1}\right)-W_{\tau_{n, i}}<\frac{1}{2} \frac{\sqrt{t}}{2^{n}}\right) \\
+ & \sum_{i=0}^{2^{2 n}-2}\left(F_{x}^{\prime}\left(\tau_{n, i+1}, \eta_{3, i}^{n}\right) \frac{\sqrt{t}}{2^{n}}-F_{x}^{\prime}\left(\tau_{n, i+1}, \eta_{4, i}^{n}\right) \frac{\sqrt{t}}{2^{n}}\right) \\
& \times I\left(0 \leqslant W_{\tau_{n, i}}+\frac{\sqrt{t}}{2^{n}}-g\left(\tau_{n, i+1}\right)<\frac{1}{2} \frac{\sqrt{t}}{2^{n}}\right) .
\end{aligned}
$$

Обозначим $\varepsilon:=\left(\sqrt{t} / 2^{n+1}\right) n$, тогда последнее выражение примет вид

$$
\begin{gathered}
\sum_{i=0}^{2^{2 n}-2}\left(F_{x}^{\prime}\left(\tau_{n, i+1}, \eta_{1, i}^{n}\right)-F_{x}^{\prime}\left(\tau_{n, i+1}, \eta_{2, i}^{n}\right)\right) \frac{1}{4 \varepsilon} I\left(0 \leqslant g\left(\tau_{n, i+1}\right)-W_{\tau_{n, i}}<\varepsilon\right) \frac{t}{2^{2 n}} \\
+\sum_{i=0}^{2^{2 n}-2}\left(F_{x}^{\prime}\left(\tau_{n, i+1}, \eta_{3, i}^{n}\right)-F_{x}^{\prime}\left(\tau_{n, i+1}, \eta_{4, i}^{n}\right)\right) \\
\times \frac{1}{4 \varepsilon} I\left(0 \leqslant W_{\tau_{n, i}}+\frac{\sqrt{t}}{2^{n}}-g\left(\tau_{n, i+1}\right)<\varepsilon\right) \frac{t}{2^{2 n}} .
\end{gathered}
$$

Так как из каждой «критической» четверки мы выбираем одну тройку, то хотя бы один из индикаторов будет равен нулю. Поэтому 
введем пару $\eta_{5, i}^{n}$ и $\eta_{6, i}^{n}$ - равную либо паре $\eta_{1, i}^{n}, \eta_{2, i}^{n}$, либо паре $\eta_{3, i}^{n}, \eta_{4, i}^{n},-$ для которой индикатор не равен нулю (в случае, если оба индикатора равны нулю, можно выбрать любую пару).

Используя новые обозначения, перепишем последнее выражение в виде

$$
\sum_{i=0}^{2^{2 n}-2}\left(F_{x}^{\prime}\left(\tau_{n, i+1}, \eta_{5, i}^{n}\right)-F_{x}^{\prime}\left(\tau_{n, i+1}, \eta_{6, i}^{n}\right)\right) \frac{1}{4 \varepsilon} I\left(\left|W_{\tau_{n, i}}-g\left(\tau_{n, i+1}\right)\right|<\varepsilon\right) \frac{t}{2^{2 n}} .
$$

Рассуждения, аналогичные рассуждениям для суммы по $U_{5}^{n}$ позволяют заменить предел этого выражение пределом следующего:

$$
\sum_{i=0}^{2^{2 n}-2}\left(F_{x}^{\prime}\left(\tau_{n, i+1}, \eta_{5, i}^{n}\right)-F_{x}^{\prime}\left(\tau_{n, i+1}, \eta_{6, i}^{n}\right)\right) \frac{1}{4 \varepsilon} I\left(\left|W_{\tau_{n, i}}-g\left(\tau_{n, i+1}\right)\right|<\varepsilon\right)\left(\tau_{n, i+1}-\tau_{n, i}\right) .
$$

Разбиваем на две отдельные суммы и устремляем $n$ к бесконечности. Каждая из них будет стремиться к интегралу, аналогичному локальному времени $\left(L_{t}=\lim _{\varepsilon \downarrow 0}(2 \varepsilon)^{-1} \int_{0}^{t} I\left(\left|W_{s}\right| \leqslant \varepsilon\right) d s\right)$. При этом $F_{x}^{\prime}\left(\tau_{n, i+1}, \eta_{5, i}^{n}\right)$ и $F_{x}^{\prime}\left(\tau_{n, i+1}, \eta_{6, i}^{n}\right)$ перейдут соответственно в пределы производной справа и слева.

Таким образом, мы получили, что «оставшиеся» слагаемые третьей суммы стремятся $\mathrm{K}$

$$
\lim _{\varepsilon \downarrow 0} \frac{1}{2 \varepsilon} \int_{0}^{t} \frac{F_{x+}^{\prime}(s, g(s))-F_{x-}^{\prime}(s, g(s))}{2} I\left(\left|W_{s}-g(s)\right|<\varepsilon\right) d s,
$$

что вместе с предыдущими рассуждениями доказывает нашу формулу. Теорема 1 доказана.

Следствием теоремы 1 является формула Танака (предложение 2).

\section{3. Случай разрывной функции.}

Теорема 2. Пусть монотонная функиия $g \in C^{1}, g(0) \neq 0$, является границей множества $A$ в $\mathbf{R}^{2} u$

$$
F(t, x)=\left\{\begin{array}{l}
f(t, x) \in C^{1,2}(\bar{A}) \\
\phi(t, x) \in C^{1,2}\left(\overline{\mathbf{R}^{+} \times \mathbf{R} \backslash A}\right) .
\end{array}\right.
$$

Тогда

$$
\begin{aligned}
& F\left(t, W_{t}\right)-F(0,0)=\int_{0}^{t} \frac{\partial F}{\partial t}\left(s, W_{s}\right) I\left(W_{s} \neq g(s)\right) d s \\
& \quad+\int_{0}^{t} \frac{\partial F}{\partial x}\left(s, W_{s}\right) I\left(W_{s} \neq g(s)\right) d W_{s}+\frac{1}{2} \int_{0}^{t} \frac{\partial^{2} F}{\partial x^{2}}\left(s, W_{s}\right) d s \\
& \quad+\lim _{\varepsilon \downarrow 0} \frac{1}{\varepsilon} \int_{0}^{t}\left(F_{(+)}(s, g(s))-F_{(-)}(s, g(s))\right) I\left(0<g^{-1}\left(W_{s}\right)-s<\varepsilon\right) d s
\end{aligned}
$$




$$
\begin{aligned}
& +\lim _{\varepsilon \downarrow 0} \frac{1}{\varepsilon} \int_{0}^{t} \frac{F_{+}(s, g(s))-F_{-}(s, g(s))}{2} I\left(\left|W_{s}-g(s)\right|<\varepsilon\right) d W_{s} \\
& +\lim _{\varepsilon \downarrow 0} \frac{1}{2 \varepsilon} \int_{0}^{t} \frac{F_{x+}^{\prime}(s, g(s))-F_{x-}^{\prime}(s, g(s))}{2} I\left(\left|W_{s}-g(s)\right|<\varepsilon\right) d s
\end{aligned}
$$

при существовании последних трех пределов.

Здесь и далее:

$$
\begin{array}{ll}
F_{+}(s, g(s))=\lim _{t \rightarrow s+0} F(t, g(s)), & F_{-}(s, g(s))=\lim _{t \rightarrow s-0} F(t, g(s)), \\
F_{(+)}(s, g(s))=\lim _{x \rightarrow g(s)+0} F(s, x), & F_{(-)}(s, g(s))=\lim _{x \rightarrow g(s)-0} F(s, x) .
\end{array}
$$

Д о к а з а т е л ь с т в о построено аналогично доказательству теоремы 1. Преобразования третьего члена суммы (7) - те же, что были выполнены в ходе доказательства теоремы 1 . Отличия будут в разборе двух первых членов суммы (7). Разобьем каждый член на две суммы, аналогично предыдущему доказательству $\left(U_{1}^{n}\right.$ и $U_{2}^{n}, U_{3}^{n}$ и $\left.U_{4}^{n}\right)$. При рассмотрении сумм по $U_{1}^{n}$ и $U_{3}^{n}$ единственное изменение заключается в том, что надо воспользоваться равномерной непрерывностью не на всем отрезке $[0, t]$, а на каждой из областей непрерывности отдельно. Основное отличие - в разборе сумм по $U_{2}^{n}$ и $U_{4}^{n}$.

Рассмотрим

$$
\sum_{U_{2}^{n}}\left(F\left(\tau_{n, i+1}, W_{\tau_{n, i}}\right)-F\left(\tau_{n, i}, W_{\tau_{n, i}}\right)\right)
$$

(напомним, что $U_{2}^{n}$ обозначает множество тех $i$, для которых отрезок, соединяющий точки $\left(\tau_{n, i+1}, W_{\tau_{n, i}}\right)$ и $\left(\tau_{n, i}, W_{\tau_{n, i}}\right)$, пересекает границу $\left.g(t)\right)$.

Перепишем (11) в виде

$$
\begin{aligned}
\sum_{U_{2}^{n}} & \left(F\left(\tau_{n, i+1}, W_{\tau_{n, i}}\right)-F\left(\tau_{n, i}, W_{\tau_{n, i}}\right)\right) \\
= & \sum_{i=0}^{2^{2 n}-1}\left(F\left(\tau_{n, i+1}, W_{\tau_{n, i}}\right)-F\left(\tau_{n, i}, W_{\tau_{n, i}}\right)\right) I\left(\tau_{n, i}<g^{-1}\left(W_{\tau_{n, i}}\right)<\tau_{n, i+1}\right) \\
= & \sum_{i=0}^{2^{2 n}-1}\left(F\left(\tau_{n, i+1}, W_{\tau_{n, i}}\right)-F\left(\tau_{n, i}, W_{\tau_{n, i}}\right)\right) \\
& \quad \times I\left(0<g^{-1}\left(W_{\tau_{n, i}}\right)-\tau_{n, i}<\tau_{n, i+1}-\tau_{n, i}\right) \frac{1}{\tau_{n, i+1}-\tau_{n, i}}\left(\tau_{n, i+1}-\tau_{n, i}\right) .
\end{aligned}
$$

Положим $\varepsilon=\tau_{n, i+1}-\tau_{n, i}$ и разобьем сумму на разность двух:

$$
\sum_{i=0}^{2^{2 n}-1}\left(F\left(\tau_{n, i+1}, W_{\tau_{n, i}}\right)\right) I\left(0<g^{-1}\left(W_{\tau_{n, i}}\right)-\tau_{n, i}<\varepsilon\right) \frac{1}{\varepsilon}\left(\tau_{n, i+1}-\tau_{n, i}\right)
$$




$$
\sum_{i=0}^{2^{2 n}-1}\left(F\left(\tau_{n, i}, W_{\tau_{n, i}}\right)\right) I\left(0<g^{-1}\left(W_{\tau_{n, i}}\right)-\tau_{n, i}<\varepsilon\right) \frac{1}{\varepsilon}\left(\tau_{n, i+1}-\tau_{n, i}\right) .
$$

Перейдем к пределу. В силу равномерной непрерывности $F$ на каждой из областей непрерывности можно перейти от $F\left(\tau_{n, i+1}, W_{\tau_{n, i}}\right)$ к $F_{(+)}\left(g^{-1}\left(W_{\tau_{n, i+1}}\right), W_{\tau_{n, i}}\right)$, а от $F\left(\tau_{n, i}, W_{\tau_{n, i}}\right)$ к $F_{(-)}\left(g^{-1}\left(W_{\tau_{n, i+1}}\right), W_{\tau_{n, i}}\right)$ (надо воспользоваться интегрируемостью интеграла типа локального времени). Таким образом, переходя к пределу по $n$ и суммируя полученные пределы, получаем, что выражение (11) при $n$, стремящемся к бесконечности, стремится к

$$
\lim _{\varepsilon \downarrow 0} \frac{1}{\varepsilon} \int_{0}^{t}\left(F_{(+)}(s, g(s))-F_{(-)}(s, g(s))\right) I\left(0<g^{-1}\left(W_{s}\right)-s<\varepsilon\right) d s .
$$

Осталось рассмотреть остаток второго слагаемого в (7). Мы можем записать его как интеграл от следующего выражения:

$$
\sum_{U_{4}^{\prime n}} \frac{F\left(\tau_{n, i+1}, W_{\tau_{n, i}}+\sqrt{t} / 2^{n}\right)-F\left(\tau_{n, i+1}, W_{\tau_{n, i}}-\sqrt{t} / 2^{n}\right)}{2 \sqrt{t} / 2^{n}} I_{\left(\tau_{n, i}, \tau_{n, i+1}\right]}(s) .
$$

(Здесь старое $U_{4}^{n}$ заменено множеством $U_{4}^{\prime n}$ тех $i$, для которых кривая $g$ пересекает дальнюю стенку квадрата.) Таким образом, мы получаем сумму

$$
\begin{aligned}
& \sum_{i=0}^{2^{2 n}-1} \frac{F\left(\tau_{n, i+1}, W_{\tau_{n, i}}+\sqrt{t} / 2^{n}\right)-F\left(\tau_{n, i+1}, W_{\tau_{n, i}}-\sqrt{t} / 2^{n}\right)}{2 \sqrt{t} / 2^{n}} I_{\left(\tau_{n, i}, \tau_{n, i+1}\right]}(s) \\
& \quad \times I\left(W_{\tau_{n, i}}-\frac{\sqrt{t}}{2^{n}}<g\left(\tau_{n, i+1}\right)<W_{\tau_{n, i}}+\frac{\sqrt{t}}{2^{n}}\right) .
\end{aligned}
$$

Разобьем эту сумму на две части. Рассмотрим, например, первую:

$$
\begin{gathered}
\sum_{i=0}^{2^{2 n}-1} \frac{F\left(\tau_{n, i+1}, W_{\tau_{n, i}}+\sqrt{t} / 2^{n}\right)}{2 \sqrt{t} / 2^{n}} I_{\left(\tau_{n, i}, \tau_{n, i+1}\right]}(s) \\
I\left(W_{\tau_{n, i}}-\frac{\sqrt{t}}{2^{n}}<g\left(\tau_{n, i+1}\right)<W_{\tau_{n, i}}+\frac{\sqrt{t}}{2^{n}}\right) .
\end{gathered}
$$

Здесь мы также будем пользоваться неравенством Иенсена и изометрией интеграла Ито, так что при равномерной сходимости подынтегрального выражения можно заменять выражение близким ему (в смысле равномерной сходимости).

Далее перейдем от $F\left(\tau_{n, i+1}, W_{\tau_{n, i}}+\sqrt{t} / 2^{n}\right)$ к $F_{+}\left(\tau_{n, i+1}, g\left(\tau_{n, i+1}\right)\right)$ (разность будет стремиться к нулю, данная процедура уже была проделана выше). В результате последняя сумма в примет вид

$$
\sum_{i=0}^{2^{2 n}-1} \frac{F_{+}\left(\tau_{n, i+1}, g\left(\tau_{n, i+1}\right)\right)}{2 \sqrt{t} / 2^{n}} I_{\left(\tau_{n, i}, \tau_{n, i+1}\right]}(s) I\left(\left|g\left(\tau_{n, i+1}\right)-W_{\tau_{n, i}}\right|<\frac{\sqrt{t}}{2^{n}}\right) .
$$


В силу равномерной непрерывности $F_{+}(s)$ можно перейти от $F_{+}\left(\tau_{n, i+1}, g\left(\tau_{n, i+1}\right)\right)$ к $F_{+}(s, g(s))$.

Таким образом, последняя сумма может быть записана в виде

$\int_{0}^{\tau_{n, 2^{2 n}}} F_{+}(s, g(s))\left(\sum_{i=0}^{2^{2 n}-1} \frac{I\left(\left|g\left(\tau_{n, i+1}\right)-W_{\tau_{n, i}}\right|<\sqrt{t} / 2^{n}\right)}{2 \sqrt{t} / 2^{n}} I_{\left(\tau_{n, i}, \tau_{n, i+1}\right]}(s)\right) d W_{s}$.

Отсюда получаем, что требуемая сумма стремится к

$$
\lim _{\varepsilon \downarrow 0} \frac{1}{\varepsilon} \int_{0}^{t} \frac{F_{+}(s, g(s))-F_{-}(s, g(s))}{2} I\left(\left|W_{s}-g(s)\right|<\varepsilon\right) d W_{s} .
$$

Теорема 2 доказана.

\section{СПИСОК ЛИТЕРАТУРЫ}

1. Fujita T., Kawanishi $Y$. A Proof of Itô's formula using discrete Itô's formula. Studia Sci. Math. Hungar., 2008, v. 45, № 2, p. 125-134.

2. Peskir G. A change-of-variable formula with local time on curves. - J. Theoret. Probab., 2005, v. 18, № 3, p. 499-535.

3. Peskir G., Shiryaev A. Optimal Stopping and Free-Boundary Problems. - Basel: Birkhäuser, 2006, 500 p.

4. Follmer H., Protter P., Shiryayev A.N. Quadratic covariation and an extension of Itô's formula. - Bernoulli, 1995, v. 1, № 1-2, p. 149-169.

5. Cherny A.S. Principal values of the integral functionals of Brownian motion: existence, continuity, and an extension of Itô's formula. - Lecture Notes in Math., 2001, v. 1755 , p. $348-370$.

6. Ghomrasni R., Peskir G. Local time-space calculus and extensions of Itô's formula. High Dimensional Probability III (Sandbjerg 2002). Basel: Birkhäuser, 2003, p. 177192. (Progr. Probab., p. 55.)

7. Itô K. Stochastic integral. - Proc. Imp. Acad. Tokyo, 1944, v. 20, p. 519-524.

8. Tanaka $\mathrm{H}$. Note on continuous additive functionals of the 1-dimensional Brownian path. - Z. Wahrscheinlichkeitstheor. verw. Geb., 1963, v. 1, p. 251-257.

9. Bouleau N., Yor M. Sur la variation quadratique des temps locaux de certaines semimartingales. - C.R. Acad. Sci. Paris, 1981, v. 292, № 9, p. 491-494.

10. Eisenbaum $N$. Integration with respect to local time. - Potential Anal., 2000, v. 13, № 4 , p. 303-328. 